3. Javaloyas N, Admetlla M, Closas J, García MD. Absceso de pulmón por Streptococcus pneumoniae. Med Clin (Barc) 1989; 93: 479.

4. Gransden WR, Eykyn SJ. Empyema in pneumococcal bacteremia. Rev Infect Dis 1988; 10: 1227-8.

\section{Metástasis mamaria de carcinoma gástrico simulando un carcinoma inflamatorio}

\section{Sr. Director:}

Cuando ocurre diseminación metastásica por vía sanguínea en pacientes con carcinoma gástrico, el lugar de asiento más frecuente es el hígado, seguido de los huesos y el pulmón. Si presenta extensión directa a través de la pared gástrica, puede alcanzar los tejidos perigástricos y posteriormente pueden afectarse estructuras adyacentes como el páncreas, colon e hígado (1).

Las metástasis en la mama de una tumoración extramamaria son muy poco frecuentes, describiéndose una incidencia del 1,2 al 2,2\% en casos clínicos y alcanzando hasta el $2,7 \%$ en series citológicas (2).

Las metástasis en la mama del cáncer gástrico son excepcionales y hasta este momento se han comunicado 23 casos en la literatura, alguno de ellos presentado en revistas muy poco accesibles y únicamente revisadas mediante Abstract en el Medline (2-8).

En estos casos, puede simular una tumoración primaria de la mama, así se observará una lesión palpable generalmente localizada en un cuadrante, aunque en ocasiones las lesiones pueden ser bilaterales y difusas simulando un carcinoma inflamatorio de mama, que tiene una incidencia de 1-2\% de todos los cánceres de la mama y clínicamente se caracteriza por un cuadro de calor local, dolor e inflamación, con un aspecto rojizo de la mama, que se puede acompañar o no de una tumoración palpable y simular a una mastitis. Histológicamente se observará una infiltración de los vasos linfáticos de la piel por un carcinoma acompañado de edema dérmico y dilatación vascular (9). malo.

El pronóstico del carcinoma inflamatorio de la mama es muy

En la mamografía será poco frecuente la observación de lesiones espiculadas y la existencia de microcalcificaciones, al contrario de lo que existe en los casos de carcinoma primario de la $\operatorname{mama}(4,5)$

Realizar la identificación correcta de una lesión metastásica en la mama respecto a un carcinoma primario de la mama resultará una medida fundamental para el manejo posterior de la paciente, obviando la realización de cirugías innecesarias y teniendo en cuanta además las diferencias en el tratamiento quimioterápico de los cánceres de mama y del estómago.

Hace algunos años tuvimos ocasión de diagnosticar un caso de metástasis mamaria de un carcinoma gástrico y nos parece interesente presentar el caso por su rareza.

Mujer de 63 años de edad, con antecedentes personales de gastrectomía subtotal 5 años atrás por un carcinoma mucosecretor bien diferenciado con células en anillo de sello. No se remitió a oncología, siguiendo revisiones en cirugía.

A los cinco años de la intervención presentó una tumefacción del miembro superior izquierdo, realizándose una flebografía en la que se observaron datos de trombosis subaguda de la vena axilar izquierda, con desarrollo de circulación colateral. Además existía una trombosis de la vena cefálica. Recibió tratamiento con heparina de bajo peso molecular y posteriormente dicumarínicos con evolución clínica inicialmente favorable.

Dos meses más tarde presentó un aumento del tamaño y de la temperatura de la mama izquierda. El aspecto de la mama sugería fuertemente un carcinoma inflamatorio.
Una mamografía mostró un aumento de densidad en la mama izquierda con engrosamiento cutáneo y afectación axilar homolateral, sugestivo de carcinoma inflamatorio.

Se realizó una biopsia, observándose la existencia de permeación linfática por un carcinoma mucosecretor con células en anillo de sello, los receptores hormonales fueron negativos.

La histología era exactamente igual a la del carcinoma gástrico intervenido anteriormente.

Laboratorio: hemograma, bioquímica, Ca 15.3 y CEA normales.

Se realizó una gastroscopia, en la que se observó un muñón gástrico sin lesiones.

TAC torácico: patrón intersticial bilateral sugestivo de linfangitis carcinomatosa, además existía un derrame pleural bilateral en moderada cuantía.

TAC abdominal: no se observaron metástasis hepáticas ni adenopatías de tamaño patológico.

Fue diagnosticada de metástasis en mama y pulmón de carcinoma gástrico, realizándose quimioterapia con esquema: etoposido, 5-Fluorouracilo, así como ácido folínico.

Se administrándose dos ciclos, sin obtener respuesta. La evolución fue rápidamente desfavorable, teniendo un pésimo estado general, por lo que se desestimaron nuevas líneas de quimioterapia, falleciendo a los dos meses del diagnóstico de la enfermedad metastásica.

\section{F. Marcos Sánchez, M. I. Albo Castaño, A. Viana Alonso, S. Casallo Blanco, F. Juárez Ucelay}

Servicio de Medicina Interna. Hospital Nuestra Señora del Prado. Talavera de la Reina. Toledo

1. Thompson GB, Van Hereden JA, Sarr MG. Adenocarcinoma of the stomach: are we making any progress?. Lancet $1993 ; 342: 713-718$.

2. Di Cosimo S, Ferreti G, Fazio N, Mandala M, Curigliano G, Bosari S et al. Breast and ovarian metastatic localization of signet ring cell gastric carcinoma. Ann Oncol 2003; 14: 803-804.

3. Hamby LS, McGrath PC, Cilbull ML, Schwartz RW. Gastric carcinoma metastatic to the breast. J Surg Oncol 1991; 48: 117-12.

4. Alexander HR, Turnbull AD, Rosen PP. Isolated breast metastases from gastrointestinal carcinomas: Report of two cases. J Surg Oncol 1989; 42 264-266.

5. Kwak JY, Kim EK, Oh KK. Radiologic findings of metastatic signet ring cell carcinoma to the breast from stomach. Yonsei Med J 2000; 41: 669-672.

6. Park JM, Kwon JS, Gong G. Metastatic breast carcinoma from gastric cancer: A case report. J Korean Radiol Soc 1998; 38: 1139-1141.

7. Domanski HA. Metastases to the breast from extramammary neoplasms. A report of six cases with diagnosis by fine needle aspiration cytology. Acta Cytol 1996; 40: 1293-1300.

8. Friedrich T, Kellermann S, Leinung S. Atypische Metastasierung eines Magenkarzinoms. Zentralbl Chir 1997; 122: 117-121 (Abstract).

9. Pérez Manga G. Carcinoma inflamatorio de la mama. En: Cáncer de mama de Pérez Manga G. Madrid: Ediciones MCR 1989; 80.

\section{Pseudoaneurisma de arteria esplénica como complicación de pancreatitis}

\section{Sr. Director:}

Los pseudoaneurismas de arteria esplénica (PAE) son una rara pero catastrófica complicación de diversas situaciones como la pancreatitis, los traumatismos abdominales, la úlcera péptica, las complicaciones post-quirúrgicas, siendo en un considerable número idiopáticos $(1,2)$. Se han descrito sólo 157 casos en la literatura, de los cuales el $50 \%$ aproximadamente ocurren en el 
contexto de pancreatitis (3). Esta complicación puede provocar sangrado gastrointestinal masivo y necesita de un manejo médico-quirúrgico específico, por lo que debe mantenerse un alto índice de sospecha para la misma (4). La oportunidad de estudiar un paciente con dicho cuadro clínico nos motiva a esta publicación.

Paciente varón de 35 años con antecedentes de ingesta elevada de alcohol y un episodio de pancreatitis aguda cuatro años antes, que ingresa por dolor en epigastrio irradiado a hipocondrio izquierdo y espalda. En la radiografía de abdomen se observan calcificaciones en la región pancreática y en la tomografía computarizada (TC) datos compatibles con pancreatitis aguda y pseudoquiste pancreático próximo a hilio esplénico. Durante el ingreso persisten las molestias en hipocondrio izquierdo, por lo que se mantiene reposo intestinal con nutrición parenteral y antibioterapia. En la TC de control realizada a los 15 días de la primera se evidencia imagen compatible con PAE, que se confirma mediante arteriografía (Fig. 1.a y 1.b ). Se intentó la embolización, siendo imposible su cateterización. El paciente fue intervenido quirúrgicamente de forma programada 15 días después, sin tener evidencia de sangrado, realizándose esplenectomía. El postopera-

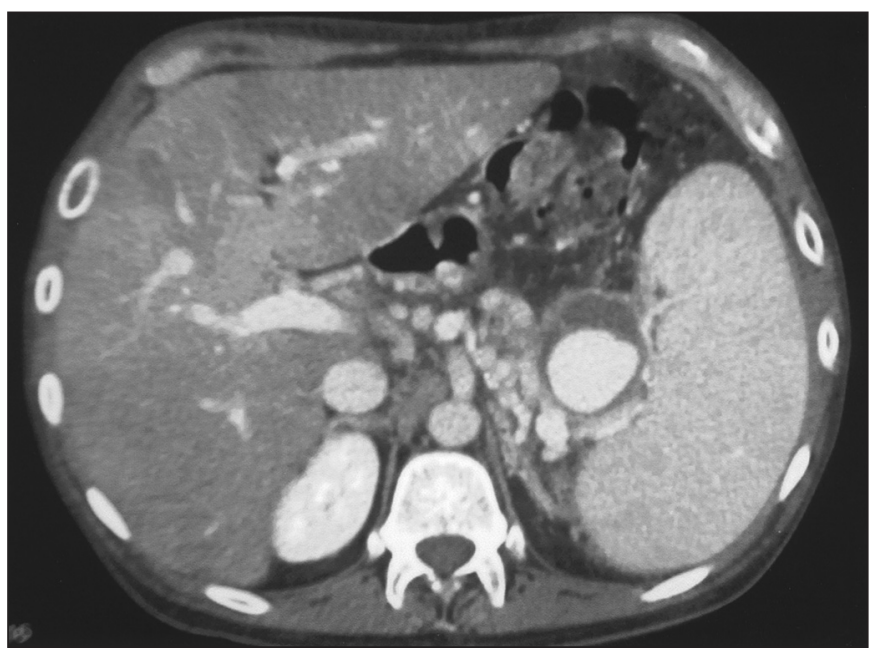

Fig. 1a: TC abdominal donde se aprecia imagen nodular paraesplénica compatible con pseudoaneurisma.

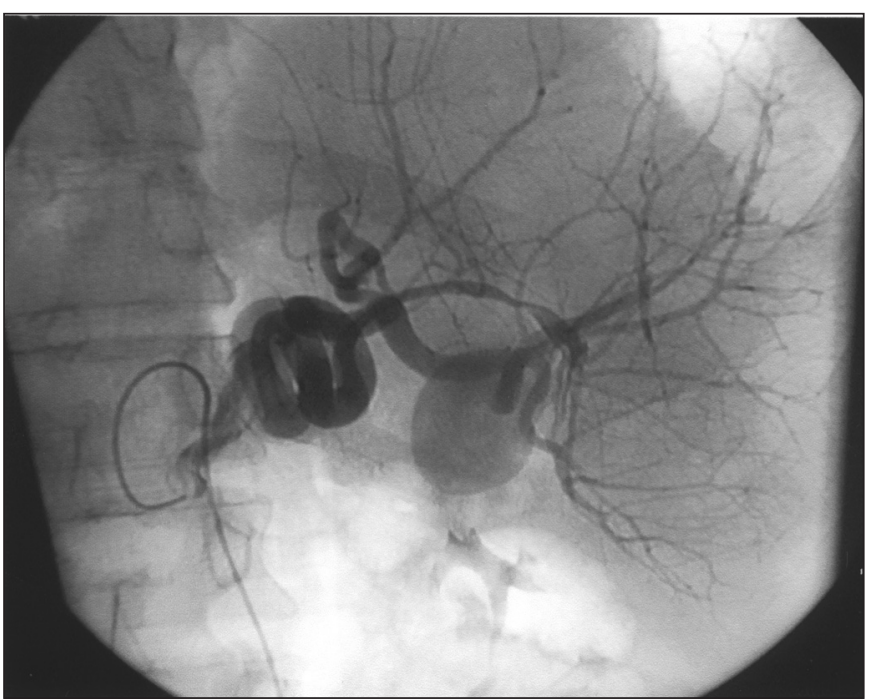

Fig. 1b. arteriografía de arteria esplénica en la que se observa el pseudoaneurisma de arteria esplénica. torio cursó sin complicaciones. El estudio histopatológico mostró pieza de esplenectomía con un área cavitada de $3,5 \times 3 \mathrm{~cm}$ en región hiliar, en relación con dilatación aneurismática de arteria esplénica. A los 10 días, estando todavía con nutrición parenteral, el paciente presentó cuadro de shock séptico fulminante por Klebsiella pneumoniae, falleciendo en la unidad de cuidados intensivos.

El mecanismo de formación del pseudoaneurisma ha sido atribuído a la digestión de la pared arterial por parte de las enzimas pancreáticas y en ocasiones a la rotura de los pseudoquistes pancreáticos $(3,5)$. En nuestro paciente se apreció en la TC abdominal del ingreso pseudoquiste pancreático, por lo que pensamos que la aparición posterior del pseudoaneurisma puede tener relación con la presencia del mismo. La clínica varía desde un hallazgo incidental, como ocurrió en nuestro caso, hasta un shock hemodinámico secundario a la rotura del pseudoaneurisma (3). La hemorragia puede provocar un sangrado masivo en cavidad peritoneal, órganos adyacentes o conductos pancreáticos, no determinando el tamaño del pseudoaneurisma el riesgo de rotura (6). El diagnóstico se realiza por TC, aunque la técnica más útil y comunmente utilizada es la arteriografía, que además tiene los beneficios de la embolización en pacientes apropiados (7). En nuestro caso fue necesario realizar arteriografía para confirmar el diagnóstico, siendo imposible la embolización al no poder cateterizarse la arteria esplénica. El tratamiento puede ser conservador, quirúrgico o embolización transcatéter (8). La cirugía consiste, según el caso, en esplenectomía con o sin pancreatectomía distal o la ligadura simple (3). Los pseudoquistes de gran tamaño asociados a pseudoaneurisma son subsidiarios de tratamiento quirúrgico debido a la dificultad de la embolización en las grandes cavidades de los pseudoquistes (3), como en el caso que presentamos.

\section{A. B. Argibay Filgueira, B. Maure Noia, P. Lamas Domín- guez, C. Martínez-Vázquez}

Servicio de Medicina Interna. Hospital Xeral-Cíes. Complejo Hospitalario Universitario de Vigo. Universidad de Santiago de Compostela

1. Srinivas M, Katavia R, Bhatnagar V, Tandon N, Iyer KV,Gupta AK. Intra-pancreatic splenic artery pseudoaneurysm: a rare complication of cronic calcific pancreatitis. Pediatric Surg Int 1998; 13: 171-4.

2. Sugg SL, Gerndt SJ, Halminton BJ, Francis IR, Taberi PA, Rodríguez JL. Psedoaneurysms of the intra parenchymal splenic artery after blunt abdominal trauma: a complication of nonoperative therapy and its management. J Trauma 1995; 39: 593-5.

3. Deron J Fessier, William M Stone, Richard J Fowl, Maher A Abbas, James C Andrews, Thomas C Bower et al. Clinical features and management of splenic artery psedoaneurysm: case series and cumulative review of literature. J Vasc Surg. 2003; 38: 969-974.

4. Nicaise N, Golzarian J, van Gansbeke D, Gremer M, Struyven J, Deviere J. Rupture of pseudoaneurysm: a cause of delayed hemorrhage after endoscopic cystoenterostomy; angiographic diagnosis and treatment. Gastrointest Endosc. 1998; 47: 186-9.

5. Golzarian J, Nicaise N, Deviere J, Ghysels M, Wery D, Dussanssois L. Transcatheter embolization of psedoaneurysm complicating pancreatitis. Cardiovas Intervent Radiol 1997; 20: 435-440.

6. Ammori BJ, Madan M, Alexander DJ. Haemorrhagic complications of pancreatitis: presentation, diagnosis and management. Ann R Coll Surg Engl 1998; 80: 316-25.

7. Vujic I, Andersen B, Stanely JH, Gobien RP. Pancreatic and peripancreatic vessels. Embolization for control of bleeding in pancreatitis. Radiology. 1984, 150. 51-2.

8. Baker KS, Tisnado J, Cho SR, Beachley MC. Splanchnic artery aneurysm and pseudoaneurysm: transcatheter embolization. Radiology. 1987; 163: 135-9. 